\title{
Training the IMG to be a clinician: The pedagogic challenge to curriculum formulators
}

\author{
N Ananthakrishnan
}

Dean of Faculty and Professor of Eminence - Surgery, Sri Balaji Vidyapeeth, Pondicherry, India

*Corresponding Author: N Ananthakrishnan

Email:

\section{Introduction}

For the first time in independent India, after a year's toil by two working groups, the product or the outcome of the undergraduate medical education system was clearly defined in the Vison 2015 document of the erstwhile Medical Council of India released in 2011 under the first set of Board of Governors. ${ }^{1}$

These desired outcome attributes of the so called Indian Medical Graduate (IMG) were:

1. Clinician, who understands and provides preventive, promotive, curative, palliative and holistic care with compassion,

2. Leader and member of the health care team and system with capabilities to collect analyse and synthesize health data,

3. Communicator with patients, families, colleagues and community.

4. Lifelong learner committed to continuous improvement of skills and knowledge.

5. Professional, who is committed to excellence, is ethical, responsive and accountable to patients, community, and profession,

Although scheduled to be implemented by 2015, the recommendations remained unnotified till December, 2017 when the MCI officially released the AETCOM module which stands for Attitude, Ethics and Communication skills for undergraduates in medicine as a curricular reform process. The full recommendations are still to be made operational.

The listed attributes of the Indian undergraduate in medicine are, however, not unique to India and closely follows the core competencies as prescribed by other countries such as USA, Canada etc. ${ }^{2,3}$ Although the phraseology and number of competencies may differ at first reading, in point of fact, they refer to the same traits as seen in the table 1.

Looking at the table, it may appear that the qualities are exclusive of each other and refer to different traits without overlap. This is not correct. If one looks back at the definition of the "Clinician" as found in the IMG document, it becomes clear that a clinician is not only one who provides clinical care but one who understands and provides preventive, promotive, curative, palliative and holistic care with compassion. This implies that not only training in Medical Knowledge and skills is required but also every other listed attribute such as professionalism, a communicator, one who keeps his knowledge updated and works within the system of health care delivery in the country.

This expanded definition, conforms to the popular perception of who is a "good doctor or clinician." A good clinician in the public's mind, in addition to be being a good diagnostician and therapist, is one whose decorum as seen by attire and attitude looks respectable and trustworthy (and hence a professional), one who can comprehend, understand and respond to patients or relatives questions (and hence a communicator) one who is competent and updated in his knowledge (and hence a medical expert), a scholar, one who believes in constant improvement of knowledge (hence practice based learning and improvement), one who is empathetic and ethical (and hence a professional) and one who understands and works within the health care system of the community and the country with his feet firmly planted on the ground. A good clinician is, therefore, one who has all the six competencies as per ACGME, the five competencies of the IMG or the seven competencies of CAN Med and not merely one who is proficient in medical knowledge and skills.

Having defined the IMG, the questions to be answered are,

1. How can the importance of being a competent and good clinician be taught and imbibed by the IMG?

2. What should the strategies adopted be at the Undergraduate and Postgraduate level?

3. How can the above be achieved within and beyond the classroom?

The degree of maturity, the time available, the prescriptive nature of the syllabus and the number of students involved dictate different approaches for the undergraduate and the postgraduate.

Table 1

\begin{tabular}{|l|c|c|}
\hline \multicolumn{1}{|c|}{ ACGME } & CAN Meds & IMG \\
\cline { 1 - 2 } Medical knowledge & Medical Expert & Clinician \\
\cline { 1 - 2 } Patient Care & Professional & Professional \\
\hline Professionalism & Communicator & Communicator \\
\hline Interpersonal and Communication skills & Scholar & Lifelong learner \\
\hline Practice based learning and improvement & Collaborator, Manager, Health Advocate & $\begin{array}{c}\text { Leader and Member of the Health } \\
\text { Care Team and system }\end{array}$ \\
\hline System Based Practice & & Tarn \\
\hline
\end{tabular}




\section{Training of undergraduate IMGs to be "a good clinician"}

The task becomes multi-dimensional in view of the expanded definition of a "good clinician." Training in the theoretical aspect of medical knowledge and requisite skills is probably the easiest to impart and is generally well taken care of in the curriculum. While being aware of the difficulties of having to deal with large numbers of students at the same time, efforts to promote more "small group activities" and problem oriented and modular teaching will help to a large extent in this process. This, however, is likely to be influenced by the nature of different medial colleges, with different numbers of student intake and faculty strength and varying physical facilities.

Skill training can be improved by greater use of skill laboratories which have now become standard requirement for medical colleges. Embedding skill training in the skill lab, in the regular curriculum, greatly facilitates this process and enables repeated practice without harming the patients. This becomes particularly important in view of inadequate patient numbers and the reluctance of patients to be seen repeatedly by students.

Communications skills training will be greatly facilitated by the requirement of early clinical exposure as and when the Vision 2015 document becomes operational. However, the available period currently allotted to community medicine in the first phase of the curriculum can be utilized for this purpose to encourage students to visit people in their home and learn to communicate in a manner that is understood by the general public.

Greater and greater use of standardized patients is an important measure to develop a good clinician communicator. Standardized patients can be anyone from the neighbouring villages who can be available at nominal financial compensation to come to the hospital and who can be trained in answering specific questions put by students. The training of standardized patients while appearing foreboding is not difficult since they have to respond to only simple questions and can be tutored to give the correct answers. This facilitates repeated practice by students without involving real patients; moreover, if it forms part of a formative OSCE process it can be used for assessing students' communication ability and giving feedback for improvement. Group activities also encourage verbal communications skills amongst peers.

Impressing on the need for constant updating of knowledge to remain a "good clinician" is a Herculean task. This habit has to be fostered during the undergraduate course by exercises such as frequent quiz programs, student seminars, small research projects and assignments.

Community exposure and outdoor activities including organising small conferences, intercollegiate competitions, sports events and cultural activities promotes leadership training. These can be supplemented by deliberate exposure to case scenarios in which the importance of the leadership role of the physician becomes exemplified.

It is in training in professionalism and ethics that maximum time and resources have to be spent. For this the importance of role modelling and mentorship programs cannot be over emphasised. However, great care must be exercised in selecting mentors. The selected mentors should be consistent in being a role model and not be prone to unprofessional behaviour even if it happens rarely. Mentorship should be voluntary and not thrust on unwilling faculty. Unprofessional mentors, even if episodic, serving as role models are likely to create distrust of the system and disbelief on the importance of professionalism in the minds of young undergraduates. Workshops in small groups, discussion on case scenarios of ethical and unethical behaviour, training in communication in delivering bad news or obtaining informed consent from patients, videos with good and bad examples facilitates this process. Providing an opportunity for clerkship during the undergraduate course wherein the undergraduate spends the whole day with the unit for a few weeks during his postings as a member of the unit and watches others in action contributes to observing and internalising some of these skills from the other members of the unit.

The effort is directed to emphasising the four D's and the four A's of a true medical professional, viz. Demeanor, Decorum, Discipline, Determination to work for the patient's welfare and Attire, Attitude, Availability for attending to patient's need at all times and Application to the patient's welfare above self.

The current curricular system does not permit a satisfactory summative assessment of these qualities. Hence reliance has to be placed on a robust formative assessment program involving standardised patients, skill labs, OSCEs, observation, peer and near peer feedback.

The effort has to be multidimensional and no single approach is likely to be entirely satisfactory. Many of the activities listed above can be carried out outside the formal curricular time. A completely competency-based training program is very difficult at the undergraduate levels considering the large numbers involved and the limited amount of faculty available.

\section{Training of postgraduates to be good IMGs}

Limited number of post-graduates per department makes training as "a good clinician" far easier in the postgraduate programs. Also small numbers enable a competency based program to be introduced as a plan of teaching and training without linking it to the summative examination process which is under regulatory control.

A good postgraduate student should have all the attributes mentioned by ACGME, CAN Med or IMG as is required for undergraduates. However, for postgraduates the standard of acceptability has to be fixed at a higher level. For this purpose the following steps need to be taken. ${ }^{4}$

1. Conversion of the syllabus and attributes of a good postgraduate to a format which is observable and measurable during the formative process. For this purpose the best method is to rewrite the qualities of the outgoing postgraduate in the form of Entrustable Professional Activities or EPAs. The list should be about 30-40 EPAs per department, in clear and 
measurable terms. This has to be done by the departmental faculty.

2. Each competency should then be analysed to see which sub-competency under ACGME list it falls in to; for example, 1. Medical knowledge, 2. Patient care skills, 3. Communication skills, 4. Opportunity for continuous improvement based on practice, 5. Professionalism and 6. Compliance with system requirements. At the postgraduate level most EPAs are likely to involve almost all these six domains. The classification of EPAs in to sub-competencies is required so that the methods of evaluation can be suitably selected to assess that particular domain.

3. Deciding on acceptable levels of performance in these domains is important. For this the Dreyfuss model which classifies competencies in to five levels as shown below is quite optimal. ${ }^{5}$ For most post-graduate courses levels four or three are acceptable.

1. Novice - is unable to perform the skills

2. Advanced Beginner - can perform under strict supervision

3. Competence - can perform with loose supervision

4. Proficiency - can perform independently

5. Expertise - can teach others

4. Fixing milestones of acceptability at the end of each six monthly period of training so that continuous monitoring, confidential feedback and opportunity to improve can be provided to those lagging behind.

5. Fixing evaluation methods for these sub-competencies which are appropriate. For postgraduates, keeping in mind the higher standards required, it may be necessary to introduce confidential multi-source feedback from peers, near-peers, supervisors, nurses, attenders, patients and relatives to give a $360^{\circ}$ view of the postgraduate performance. The feedback forms should be simple and acceptable in the cultural context merely requiring other health care workers to state whether the behaviour of the concerned postgraduate is better than expected, acceptable or falls short of acceptability as regards that particular parameter. Extreme confidentiality is absolutely a must to encourage other health care workers to give a feedback.

6. Having a system of continuous monitoring and feedback which goes beyond the conventional log book is necessary. For this purpose an e-portfolio is optimal.

Since ensuring an acceptable standard of performance is extremely important for postgraduates, who after qualification, are going to serve as specialists in their field, the process has to be constantly mentored and monitored. Small numbers of postgraduates compared to undergraduates, gives an opportunity to have more case based discussions, workshops, and other small group activities particularly on complex areas such as professionalism and ethics.

\section{Conclusion}

To sum up it may be said that the term "good clinician" as mentioned in the IMG document has far greater implication than the mere translation of the phrase in to someone who is good in diagnosis and treatment. It encompasses several skills which have to be embedded in the individual to qualify the person as a "good clinician" and unless this is made aware to all those who plan and executive the teaching learning program, efforts are likely to be directed only at improving information transfer and development of manipulative skills which is not the intention of classifying someone as a "good clinician" in the IMG document.

The best workable model will be to insist on a core set of attributes which constitutes a core curriculum and which can be initiated in the foundation course of Vision 2015 document. This can be visited repeatedly in a spiral curriculum at every stage for reemphasis.

\section{References}

1. Medical council of India, Reforms in Under-graduate and Postgraduate Medical education, Vision, 2015. https://www.tnmgrmu.ac.in/images/medical-council-ofindia/MCI_book.pdf (accessed on 14.10.2018).

2. NEJM Knowledge + team, Exploring the ACGME Core Competencies (Part 1 of 7)

https://knowledgeplus.nejm.org/blog/exploring-acgme-corecompetencies/ (accessed on 14.10.2018).

3. Royal College of Physicians and Surgeons of Canada. CanMEDS: Better standards, better physicians, better care. http://www.royalcollege.ca/rcsite/canmeds/canmeds-frameworke. (accessed on 14.10.2018).

4. Ananthakrishnan N, Karthikeyan P, Jaganmohan R, Pulimoottil DT, Ravishankar M, Adkoli BV et al. SBV model of Competency based learning and training (COBALT) for Postgraduate Education. Annals of SBV, 2017;6:5-9.

5. Dreyfus SE. The five stage model of Adult skill acquisition. Bulletin of Science Technology \& Society 2004;24:177. DOI: 10.1177/0270467604264992 